\title{
THE INFLUENCE OF MOMENTUM ADDITION ON THE ACCELERATION OF THE SOLAR AND STELLAR WINDS
}

\author{
WEN-RUI HU \\ Institute of Mechanics, Academia Sinica, Beijing, China
}

(Received 5 September, 1983)

\begin{abstract}
The influence of the momentum addition, which may be associated with the average or fluctuation transverse component of the magnetic field or others, on the acceleration the solar wind or stellar wind is studied in a local streamtube. The results show that the larger the momentum addition the stronger the acceleration of the wind. For example, if the typical transverse magnetic field is about 0.1 of the longitudinal field, the velocity of the solar wind at $1 \mathrm{AU}$ may be increased by $40 \%$. The coronal hole may be considered as a streamtube, the presence of a high stream from the coronal hole may be explained by the existence of an average or fluctuation transverse magnetic field in the streamtube. A similar conclusion may be applied to the polar region, where the velocity of the solar wind will be larger than elsewhere as if there is a transverse component of magnetic field, as well as to the stellar wind. The influence of other parameters on the acceleration of the solar wind is also discussed. From the viewpoint of the solar wind mechanism, the present paper shows that the momentum addition in the subsonic flow region can increase the velocity of the solar wind at $1 \mathrm{AU}$.
\end{abstract}

\section{Introduction}

The acceleration mechanism of solar wind is a matter of importance, not only in solar physics (see Parker, 1963; and Hundhausen, 1972) but also in astrophysics (see, for examples, Holzer, 1979; Hu, 1982b). One of the important topics in the solar wind theory is the acceleration mechanism, and one of the main concerns is the inconsistency between the smaller velocities given by theoretical models and the large velocities at 1 AU observed by satellite. The difficulty is even more serious for the high-speed streams of coronal holes. It is believed that some unaccounted energy deposition must speed up the solar wind.

We analyzed the influence of energy addition on the solar wind acceleration, and shown that the solar wind velocity at $1 \mathrm{AU}$ may be increased even if the additional energy is supplied in the region of subsonic flow (Hu, 1982a). This conclusion is applied to study the acceleration process of jet beam for the double source in radio galaxy, where the jet has larger velocity and the energy is added near the center optical galaxy in the subsonic flow ( $\mathrm{Hu}, 1982 \mathrm{~b})$. These conclusions are different from the ones of Leer and Holzer (1980), because the initial and boundary conditions are different. The purpose of the present paper is to analyze the other side of the solar wind acceleration, that is, the influence of momentum addition on the acceleration, and it shows that the solar wind velocity is increased even if the momentum addition is supplied in the subsonic region.

The momentum addition may be associated with the average or fluctuation transverse component of the magnetic field or others. The solar wind may be accelerated by local Lorentz forces if the local solar magnetic field is not a potential or force-free field. One-dimensional models are often adopted in studing the acceleration of the solar wind, 
the role of the magnetic field is only considered as a confining tube along which the solar wind flows. In this consideration, the Lorentz force is absent, and the acceleration process is solely that of pure gas dynamics. However, a Lorentz force is non-zero even in the one-dimensional model if there is a non-uniform transverse component of magnetic field in or at the flux tube. This is important because the magnetic energy is larger than the kinetic energy of the solar wind at distances of less than the solar radii, and, on the other hand, a transverse component of magnetic field is suggested by the observations in the jet beam of double source in the radio galaxy (Bridle, 1982). In the present paper, we will pay attentions on the solar wind acceleration, and the influence on the radio galaxy will be discussed elsewhere.

Weber and Davis (1967) discussed the solar wind flow near the equatorial plan including the magnetic field, their results will be different from the ones in a flux tube as discussed in the present paper. Whang and Chien (1978) analyzed the influence of the Lorentz force and Alfvénic fluctuation pressure on the high-speed stream in a streamtube. Pneuman (1971), Yeh and Pneuman (1976) solved the magnetohydrodynamic equations and obtained a globally consistent solution for the solar wind. $\mathrm{Hu}$ (1981a) analyzed the transitional features of the solar wind in the local flux tube and showed that the magnetic field has an important influence on the acceleration if twodimensional effects are included, and that, in addition to the sonic velocity, there are other two critical velocities. On the other hand, Leer and Holzer (1980) discussed the influence of a source or sink of energy and momentum on the solar wind acceleration for a fixed initial mass flux, in this case, they found that the velocities at $1 \mathrm{AU}$ are decreased or changed slightly if the additional energy or momentum are supplied in the subsonic region. The present paper will discuss the solar wind flow in a fixed flux tube, and the initial mass flux changes for different additional momentum.

If there exists, within the flux tube, a transverse magnetic field, which may be the component of either the average or fluctuation field, or both, then the velocity of the solar wind may have a transverse component. The total kinetic energy of the solar wind is the sum of the transverse and longitudinal kinetic energies, where the transverse and longitudinal directions are taken with respect to the center line of the flux tube. In the solar corona, the kinetic transverse energy may be larger if the transverse component of the magnetic field is appreciable. Since we assume that the transverse component of the magnetic field is produced in a localized current region, this transverse component, as well as the transverse component of velocity, has to decrease with increasing distance from the solar surface. Therefore, far from the solar surface, the longitudinal velocity may be larger in the case where the transverse component of the field is present than in the case without the transverse magnetic field. On the other hand, the pressure of the fluctuation field may also accelerate the flow of the solar wind in the streamtube. By using these ideas, we can explain some features of the solar wind.

In Section 2, the basic equations are analyzed. In Section 3, the flow features and the influence of the momentum addition are studied qualitatively. In Section 4 , the acceleration and high-stream flow are given quantitatively for some typical model. In the last section, we discuss implications and conclusions. 


\section{The Basic Equations}

In spherical coordinates $(r, \theta, \phi)$, the distributions of magnetic field and plasma velocity are two-dimensional - i.e.,

$$
B=\left(B_{r}, 0, B_{\phi}\right)
$$

and

$$
v=\left(v_{r}, 0, v_{\phi}\right),
$$

where the configuration of flux tube is axisymmetrical. As the flux tube is slender, the varieties of quantities in $\theta$ will be small.

The equations of solar wind in the case of one-dimensional approximation may be written as

$$
\begin{aligned}
& \rho v_{r} A=m \text { (constant), } \\
& \rho v_{r} \frac{\mathrm{d} v_{r}}{\mathrm{~d} r}=-\frac{\mathrm{d} p}{\mathrm{~d} r}-\rho \frac{G \cdot \mathscr{M}}{r^{2}}+\rho \frac{v_{\phi}^{2}}{r^{2}}-\frac{B_{\phi}}{4 \pi r} \frac{\mathrm{d} B_{\phi} r}{\mathrm{~d} r}-\frac{\mathrm{d} W_{1}}{\mathrm{~d} r}, \\
& \rho v_{\phi}^{2}=\frac{B_{\phi}^{2}}{4 \pi}, \\
& \rho\left[v_{r} \frac{\mathrm{d} e}{\mathrm{~d} r}+v_{r} p \frac{\mathrm{d}}{\mathrm{d} r}\left(\frac{1}{\rho}\right)\right]=\frac{1}{r} \frac{\mathrm{d} r^{2} q_{1}}{\mathrm{~d} r}, \\
& B_{r} A=\Phi(\text { constant }), \\
& \frac{\mathrm{d}}{\mathrm{d} r}\left[r\left(v_{\phi} B_{r}-v_{r} B_{\phi}\right)\right]=0,
\end{aligned}
$$

where $\rho, p, T$, and $e$ are, respectively, the gas density, pressure, temperature, and internal energy; $W_{1}$ is the momentum addition other than the Lorentz component; $\mathbf{q}_{1}=q_{1}(r) \mathbf{e}_{r}$ is the heat flux and is assumed to be a function of $r$ only. In the right-hand side of Equation (2.4), the last three terms associate with the momentum addition. By using the method of local expansion for small $\theta(\mathrm{Hu}, 1981 \mathrm{~b}, \mathrm{c}, 1982 \mathrm{~b})$, Equations (2.3)-(2.8) may be demonstrated from the magnetohydrodynamic equations as given in the appendix. However, the equations given above are more general.

Equation (2.4) may be written as

$$
\rho v_{r} \frac{\mathrm{d} v_{r}}{\mathrm{~d} r}=-\frac{\mathrm{d} p}{\mathrm{~d} r}-\rho \frac{G \mathscr{M}}{r^{2}}-\frac{\mathrm{d} W_{*}}{\mathrm{~d} r},
$$

where the total momentum addition is

$$
W_{*}=W_{1}+\frac{B_{\phi}^{2}}{8 \pi} .
$$


The measurements of solar wind show that there are large fluctuations of magnetic field in the solar wind and the fluctuations of thermodynamical quantities are small. If the magnetic field is denoted as the sum of average and fluctuation fields, we have

$$
\mathbf{B}=\mathbf{B}_{0}+\delta \mathbf{B}
$$

In this case, the momentum addition terms in Equation (2.9) will include the contribution from the Alfvénic fluctuation pressure, and relationship (2.10) may be rewritten as

$$
W_{*}=W_{1}+\frac{B_{\phi 0}^{2}}{8 \pi}+\frac{\left\langle\delta B_{\phi}^{2}\right\rangle}{8 \pi} ;
$$

and the last term in relationship (2.12) is the average of the fluctuation magnetic pressure. Equation (2.9) agrees with that of Belcher (1971) or Whang and Chien (1978) if term $\left\langle\delta B^{2}\right\rangle / 8 \pi$ is considered as the Alfvénic fluctuation pressure, and agrees with the result of Weber and Davis (1967) if the fluctuation terms are omitted.

Basic equations (2.3), (2.9), and (2.6) are consistent if the cross section of the streamtube and the momentum addition $W$ are given. Some simplifications of Alfvénic fluctuation pressure have been suggested (see, for examples, Hollweg, 1973; Whang and Chien, 1978). However, the determination of the fluctuation pressure is still a subject for the further research. As the purpose of the present paper is majorly to study the influence of the momentum addition on the solar wind acceleration, we will discuss a typical distribution of momentum addition $W$, and the results may shed light on the acceleration processes.

\section{The Acceleration Processes}

It is easily seen that the force associated with the momentum addition is not a simple function of thermodynamical quantities, and the energy equation cannot be integrated to give a simple energy relationship. Therefore, the acceleration process of the flow field here is much more complicated than the usual solar wind acceleration.

We define the gravitational potential

$$
\psi=-\frac{G \mathscr{M}}{r},
$$

and the heat energy addition

$$
q=\int \frac{A}{r^{2}} \frac{\mathrm{d}}{\mathrm{d} r}\left(\frac{r^{2} q_{1}}{m}\right) \mathrm{d} r .
$$

The specific energy density associated with the momentum addition may be written as

$$
W=\int \frac{1}{\rho} \frac{\mathrm{d} W_{*}}{\mathrm{~d} r} \mathrm{~d} r .
$$


Then the energy integral of Equations (2.6) and (2.9) gives

$$
e+\frac{p}{\rho}+\frac{v_{r}^{2}}{2}+\psi-q+W=E \text { (constant) }
$$

In terms of the sonic velocity $a=\sqrt{\gamma \mathscr{R} T}$, Equation (3.4) becomes

$$
\frac{a^{2}}{\gamma-1}+\frac{v_{r}^{2}}{2}+\psi-q+W=E \text {. }
$$

Equation (3.5) reduces to those of a gasdynamic usual solar wind if $W=0$.

The momentum addition $W$ may be converted into the kinetic energy of the solar wind. If the typical transverse magnetic field is $1 \mathrm{G}$ and the plasma density is $10^{-16} \mathrm{~g} \mathrm{~cm}^{-3}$, then the magnitude order of $W$ will be

$$
\left.W\right|_{r=1.1 r_{\odot}} \simeq 0.5 \times 10^{15} \mathrm{~cm}^{2} \mathrm{~s}^{-2} \text {. }
$$

We compare this order of magnitude with the kinetic energy corresponding to a velocity of $400 \mathrm{~km} \mathrm{~s}^{-1}$, that is,

$$
\left.\frac{v^{2}}{2}\right|_{r=1 \mathrm{AU}}=0.8 \times 10^{15} \mathrm{~cm}^{2} \mathrm{~s}^{-2} .
$$

It shows that the energy associated with the momentum addition is not small. Therefore, the solar wind may be accelerated to higher speed if there is suitable momentum addition, such as the transverse magnetic field, or heating source. For example, if the Alfvénic fluctuation is considered, there is a fluctuation transverse velocity

$$
\delta \mathbf{v}_{\phi}=\mp \frac{\delta \mathbf{B}_{\phi}}{\sqrt{4 \pi \rho}} ;
$$

or, by use of (2.5), the transverse magnetic energy must balanced by the transverse kinetic energy, and the rotational velocity must equal the local Alfvén velocity

$$
v_{\phi}=\frac{B_{\phi}}{\sqrt{4 \pi \rho}} .
$$

In this case, the stream line of the solar wind is helical, and so is the acceleration of the solar wind.

According to Equations (2.3), (2.6), and (2.9), the acceleration equation of the solar wind is

$$
\frac{v_{r}^{2}-a^{2}}{v_{r}} \frac{\mathrm{d} v_{r}}{\mathrm{~d} r}=\frac{a}{A} \frac{\mathrm{d} A}{\mathrm{~d} r}-\frac{G \mathscr{M}}{r}-(\gamma-1) \frac{\mathrm{d} q}{\mathrm{~d} r}-\frac{\gamma}{\rho} \frac{\mathrm{d} W}{\mathrm{~d} r} .
$$

In the above equation, the last term gives the influence of the momentum addition on the solar wind acceleration. As $W$ decreases rapidly far away from the solar surfaces, 
and there is the conversion of magnetic energy into kinetic energy of the solar wind.

With the aid of the energy integral (3.5), the equation of Mach number $M=v_{r} / a$ may be rewritten as

$$
\begin{aligned}
\frac{M^{2}-1}{M^{2}} \frac{\mathrm{d} M^{2}}{\mathrm{~d} r}= & \left(1+\frac{\gamma-1}{2} M^{2}\right) \frac{2}{A} \frac{\mathrm{d} A}{\mathrm{~d} r}-\frac{(\gamma+1) G \mathscr{M}}{a^{2} r^{2}}+ \\
& +\frac{1+\gamma M^{2}}{a^{2}} \frac{\mathrm{d} q}{\mathrm{~d} r}-\frac{(\gamma+1)\left[1+(\gamma-1) M^{2}\right]}{a} \frac{M A}{m} \frac{\mathrm{d} W}{\mathrm{~d} r}
\end{aligned}
$$

where the relation between $a$ and $M$ is obtained from the equation for the conservation of energy of the form

$$
\frac{\mathrm{d}}{\mathrm{d} r}\left[\left(\frac{1}{\gamma-1}+\frac{M^{2}}{2}\right) a^{2}-\frac{G \mathscr{M}}{r}-q\right]+\frac{a M A}{m} \frac{\mathrm{d} W}{\mathrm{~d} r}=0 .
$$

Equation (3.12) may be alternatively rewritten as

$$
\begin{aligned}
\frac{M^{2}-1}{\gamma-1} \frac{\mathrm{d} a^{2}}{\mathrm{~d} r}= & -\frac{M^{2} a^{2}}{A} \frac{\mathrm{d} A}{\mathrm{~d} r}+\frac{2-(\gamma-1) M^{2}}{2+(\gamma-1) M^{2}} \frac{G \mathscr{M}}{r^{2}}+ \\
& +\frac{\gamma(\gamma-1) M^{4}+(\gamma+1) M^{2}-2}{2+(\gamma-1) M^{2}} \frac{\mathrm{d} q}{\mathrm{~d} r}+ \\
& +\frac{\left(\gamma^{2}-1\right) M^{4}-(\gamma-1) M^{2}+2}{2+(\gamma-1) M^{2}} \frac{a M A}{m} \frac{\mathrm{d} W}{\mathrm{~d} r} .
\end{aligned}
$$

If the boundary conditions are given in the form

$$
\left.M\right|_{r=r_{0}}=M_{0},\left.\quad a\right|_{r=r_{0}}=a_{0},
$$

then the flow fields of the solar wind may be solved from Equations (3.11) and (3.13), subject to the boundary conditions (3.14).

In the case $W=0$, energy integral (3.6) is an algebraic equation, and only Equation (3.11) needs to be discussed. The accelerating feature and the characteristics of the singular points may be analyzed in the $(M, r)$-plane. In the case of $W \neq 0$, we must discuss the problem in the three-dimensional space $(M, a, r)$. The integral curves of Equations (3.11) and (3.13) are curves in three dimensions which can be projected to the plane $(M, r)$, but the characteristic concept in three dimensions is quite different from those in two-dimensional problems with $W=0$. The scheme of an integral curve in three dimensions and its projections in the $(M, r)$-plane are shown in Figure 1. There may be several singular sonic points in the $(M, r)$-plane, but these sonic points may correspond to different integral curves as the critical sonic velocities are different.

Equations (3.11) and (3.13) show that $M=1$ is the singular plane, and the relation 


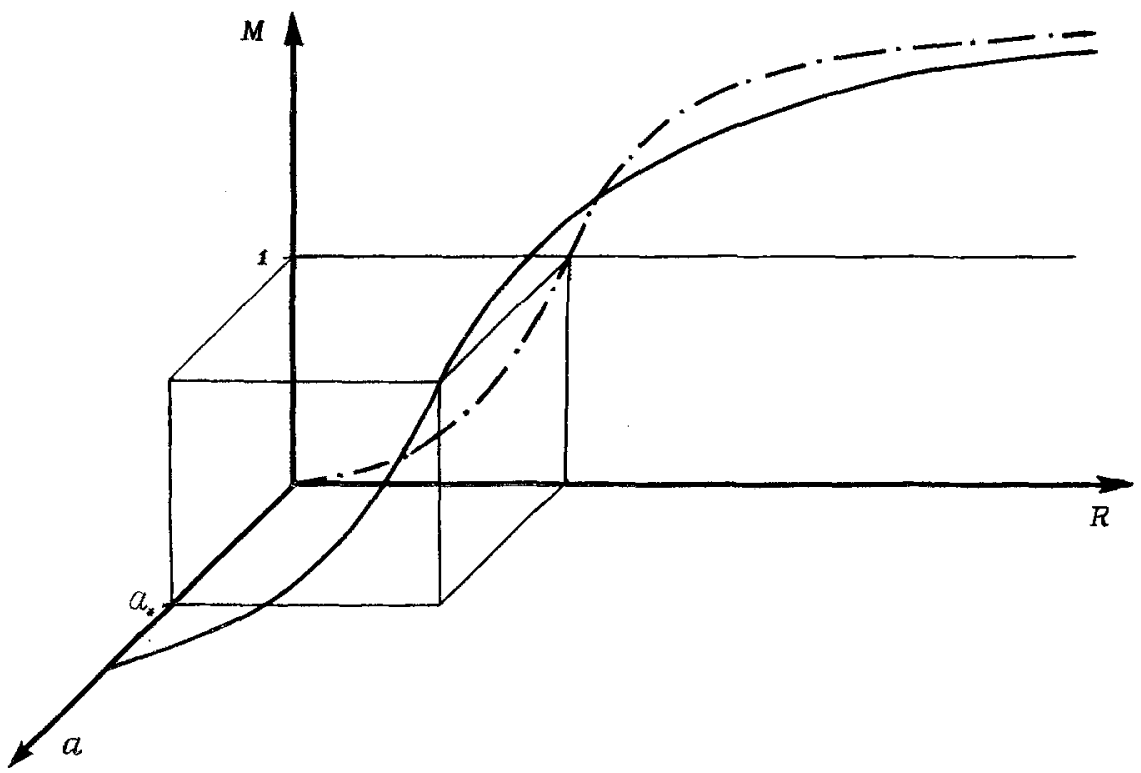

Fig. 1. The integral curve in three dimensions and its projection in plane $(M, R)$.

in this plane is

$$
\frac{\mathrm{d} \ln A\left(r_{s}\right)}{\mathrm{d} r}-\frac{1}{a_{s}^{2}} \frac{G \mathscr{M}}{r_{s}}-\frac{\gamma-1}{a_{s}^{2}} \frac{\mathrm{d} q\left(r_{s}\right)}{\mathrm{d} r}-\frac{\gamma A\left(r_{s}\right)}{a_{s} m} \frac{\mathrm{d} W\left(r_{s}\right)}{\mathrm{d} r}=0,
$$

where the critical sonic velocity and the position of sonic point are

$$
a\left(r_{s}\right)=a_{s} \text { and } \quad r=r_{s}, \quad \text { when } M=1 .
$$

By use of Equations (3.11) and (3.5), the differential relationship of the Mach number at the sonic point satisfied the equation

$$
4\left[\frac{\mathrm{d} M\left(r_{s}\right)}{\mathrm{d} r}\right]^{2}+S_{1}\left(r_{s}\right) \frac{\mathrm{d} M\left(r_{s}\right)}{\mathrm{d} r}-S_{2}\left(r_{s}\right)=0,
$$

where the coefficients are

$$
\begin{aligned}
S_{1}(r)= & 2(\gamma-1) \frac{\mathrm{d} \ln A(r)}{\mathrm{d} r}+\frac{2}{a^{2}} \frac{G \mathscr{M}}{2 r^{2}}+\frac{2\left(\gamma^{2}-1\right)}{a^{2}} \frac{\mathrm{d} q(r)}{\mathrm{d} r}+ \\
& +\frac{3 \gamma^{2}+2 \gamma-2}{m a} A(r) \frac{\mathrm{d} W(r)}{\mathrm{d} r},
\end{aligned}
$$




$$
\begin{aligned}
S_{2}(r)= & \frac{\gamma-1}{a^{4}}\left[\frac{2 G \mathscr{M}}{r^{2}}+2(\gamma-1) \frac{\mathrm{d} q(r)}{\mathrm{d} r}+\frac{\gamma A(r) a}{m}-\frac{\mathrm{d} W(r)}{\mathrm{d} r}\right] \times \\
& \times\left[\frac{\mathrm{d} q}{\mathrm{~d} r}-\frac{G \mathscr{M}}{r^{2}}-\frac{A a}{m} \frac{\mathrm{d} W}{\mathrm{~d} r}\right]+\frac{\gamma+1}{a^{2}} \times \\
& \times\left[a^{2} \frac{\mathrm{d}^{2} \ln A}{\mathrm{~d} r^{2}}+\frac{2 G \mathscr{M}}{r^{3}}-(\gamma-1) \frac{\mathrm{d}^{2} q}{\mathrm{~d} r^{2}}-\frac{\gamma(\gamma+1) a}{m} \frac{\mathrm{d}}{\mathrm{d} r}\left(A \frac{\mathrm{d} W}{\mathrm{~d} r}\right)\right] .
\end{aligned}
$$

The solutions of Equation (3.17) are

$$
\frac{\mathrm{d} M\left(r_{s}\right)}{\mathrm{d} r}=\frac{1}{4}\left[-S_{1}\left(r_{s}\right) \pm \sqrt{S_{1}^{2}\left(r_{s}\right)+16 S_{2}\left(r_{s}\right)}\right] .
$$

If the solutions give two real roots - that is, if

$$
S_{1}^{2}\left(r_{s}\right)+16 S_{2}\left(r_{s}\right)>0,
$$

there are two real characteristic directions at the sonic points, and the positive root corresponds to the integral curve along which the flow accelerates smoothly from subsonic into supersonic velocities. Projecting this three-dimensional integral curve in the $(M, r)$-plane, the sonic point is a saddle point. If the condition (3.21) is not satisfied, the flow of the solar wind cannot accelerate smoothly into the superonic one. According to the distributions of $A(r), q(r)$, and $W(r)$, there may be one or several singular points in the sonic plane $M=1$. It can be seen that the configurations of the integral curves in three dimensions are generally more complicated than that in two dimensions.

\section{The High Stream Flow}

The solar wind from the coronal hole associates with high stream flow. We analyzed the influence of the energy addition on the high stream flow ( $\mathrm{Hu}, 1982 \mathrm{a})$, and now we discuss the influence of additional momentum.

Observations show that magnetic fields in coronal holes are divergent and open, and are weaker. The dynamical process near the solar surface is easily to twist the magnetic force line in the flux tube and gives an azimuthal component of the magnetic field (see, for example, $\mathrm{Hu}, 1981 \mathrm{~b}$ ). Adopting $B_{\phi}=1 \mathrm{G}$ and $n=10^{8} \mathrm{~cm}^{-3}$, we find that the azimuthal Alfvén velocity becomes

$$
v_{\mathrm{A}}=\frac{B_{\phi}}{\sqrt{4 \pi \rho}} \simeq 200 \mathrm{~km} \mathrm{~s}^{-1} \text {. }
$$

On the other hand, the density, temperature and magnetic field in the coronal hole are smaller than those in the region outside. From the viewpoint of mechanical balance, we need some of radial forces to maintain equilibrium in the radial direction. One of these 
forces may be centrifugal force, which maintains a higher pressure in the outer region and lower pressure in the inner region.

According to (3.9), rotational velocity must exist if the azimuthal magnetic field exists, and then the solar wind is accelerated helically. The velocity of the solar wind in such a coronal hole must be larger than normal one as a larger amount of azimuthal kinetic energy is converted into the kinetic energy along the flux tube. The stronger the azimuthal magnetic field in the coronal hole, the higher the stream velocity near the Earth's orbit. Furthermore, the solar wind from the coronal hole may accelerate to high stream if other additional momentum supplies in the coronal hole.

A similar discussion may be applied to the polar region, where the magnetic field is open, and the velocity must be larger if there is a larger azimuthal component of the magnetic field or an additional momentum.

We now study the acceleration process quantitatively, and introduce the following non-dimensional quantities:

$$
\begin{aligned}
& R=\frac{r}{r_{0}}, \quad a_{1}=\frac{a}{a_{s}}, \quad A_{1}=\frac{A}{A_{s}}, \\
& G_{1}=\frac{G \mathscr{M}}{r_{0} a_{s}^{2}}, \quad \frac{q}{a_{s}^{2}}=Q_{0} f(R), \quad \frac{2 W}{a_{s}^{2}}=H_{0} g(R),
\end{aligned}
$$

where $G_{1}, Q_{0}, H_{0}$ are non-dimensional parameters, the subscription 0 denotes the initial value. We will use the following typical distributions:

$$
\begin{aligned}
& f(R)=1-\exp \left(-\frac{R-R_{0}}{R_{1}}\right), \\
& g(R)=\exp \left(-\frac{R-R_{0}}{R_{2}}\right), \\
& A_{1}(R)=R^{2}
\end{aligned}
$$

Equations (3.11) and (3.12) become

$$
\begin{aligned}
\frac{\mathrm{d} M}{\mathrm{~d} R}= & \frac{M}{2(M-1)}\left\{\frac{4}{R}\left(1+\frac{\gamma-1}{2} M^{2}\right)-\frac{(\gamma+1) G_{1}}{a_{1}^{2} R^{2}}-\right. \\
& -Q_{0} \frac{(\gamma-1)\left(1+\gamma M^{2}\right)}{a_{1}^{2} R_{1}} \exp \left(-\frac{R-R_{0}}{R_{1}}\right)+ \\
& \left.+\frac{\gamma+1}{2}\left[1+(\gamma-1) M^{2}\right] \frac{H_{0} M R^{2}}{a_{1} R_{2}} \exp \left(-\frac{R-R_{0}}{R_{2}}\right)\right\}, \\
\frac{\mathrm{d} a_{1}}{\mathrm{~d} r}= & \frac{\gamma-1}{a_{1}\left[2+(\gamma-1) M^{2}\right]}\left[M a_{1}^{2} \frac{\mathrm{d} M}{\mathrm{~d} R}+\frac{G_{1}}{R}-\frac{Q_{0}}{R_{1}} \exp \left(-\frac{R-R_{0}}{R_{1}}\right)-\right. \\
& \left.-\frac{M A_{1} H_{0}}{2 R_{2}} \exp \left(-\frac{R-R_{0}}{R_{2}}\right)\right] .
\end{aligned}
$$


If we choose the sonic conditions as

$$
M=1 \text { and } a_{1}=1 \text {, when } R=R_{s},
$$

the acceleration process of the solar wind may be solved from Equations (4.4) and (4.5). In this case, the position of sonic point is determined from condition (3.15) as

$$
R_{s}-\frac{G_{1}}{2}-\frac{(\gamma-1) Q_{0} R_{s}^{2}}{R_{1}} \exp \left(-\frac{R_{s}-R_{0}}{R_{1}}\right)+\frac{\gamma H_{0} R_{s}^{4}}{2 R_{2}} \exp \left(-\frac{R_{s}-R_{0}}{R_{2}}\right)=0 \text {, }
$$

and $R_{1}=R_{2}=1.1 R_{\odot}$ is adopted in the computations.

Figures 2 and 4 show the influence of the magnetic field on the velocity distribution. Figures 3 and 5 are the distributions of the Mach number. The parameters are adopted as $G_{1}=6.2577$ in Figures 2 and 3, and $Q_{0}=50$ and $G_{1}=2$ in Figures 4 and 5 . If the temperature at the sonic point is about $2 \times 10^{6} \mathrm{~K}$, the parameter $G_{1}=6.2577$ corresponds to the case of the Sun, a larger value of $G_{1}$ associates with a lower temperature at the sonic point, so, the value $G_{1}=2$ corresponds to the stellar wind where the star has smaller mass, higher plasma temperature and larger stellar radius. Figure 2 shows that the velocity of the solar wind at the Earth's orbit will increase about $40 \%$ if $\mathrm{H}_{0}$ increases from 0 to 0.5 . Similar conclusion about the acceleration by the momentum addition may be seen clearly from Figure 4. The distributions of the Mach number in Figures 3 and 5 show that the Mach number is increased by the increasing $H_{0}$, but the

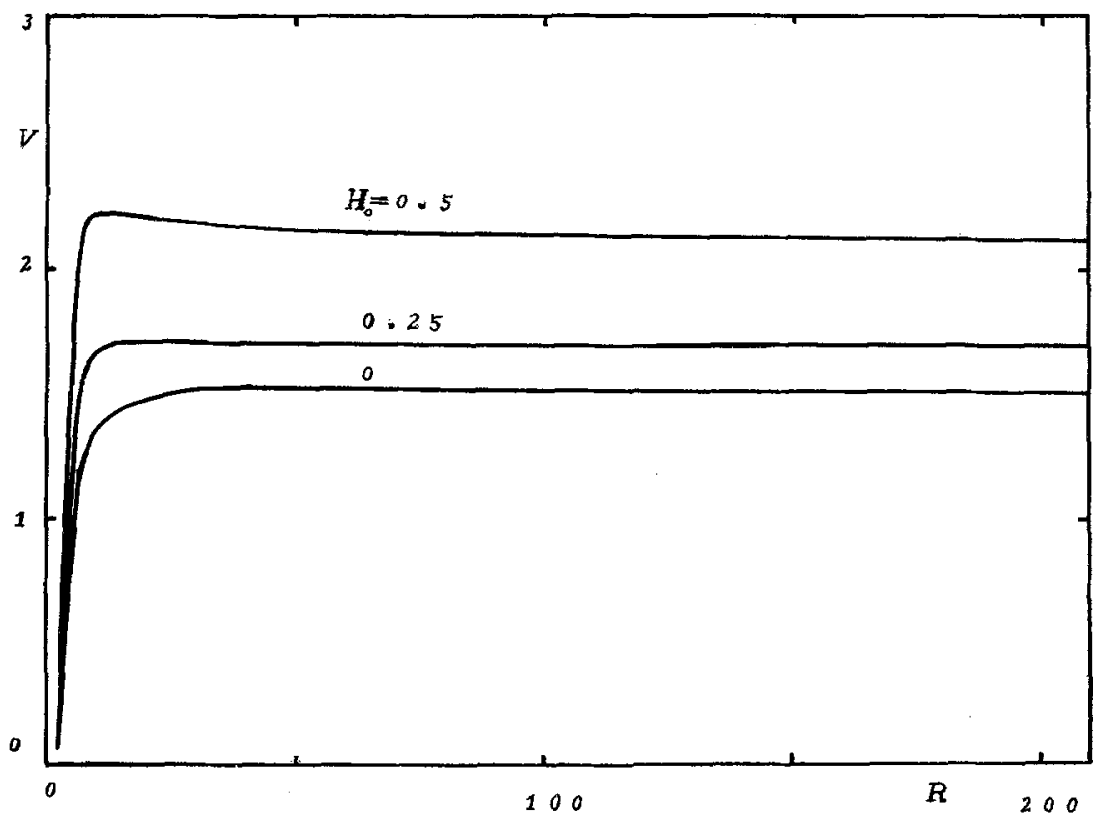

Fig. 2. The distribution of velocity, where $G_{1}=6.2577$ and $Q_{0}=15$. 


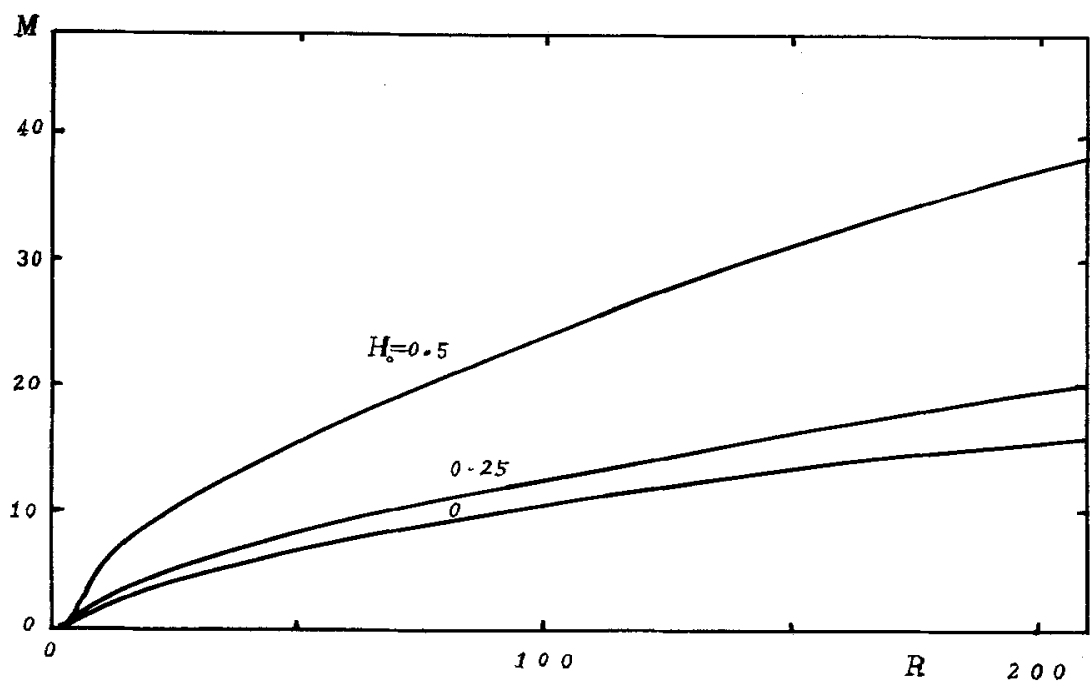

Fig. 3. The Mach number distribution, where $G_{1}=6.2577$ and $Q_{0}=15$.

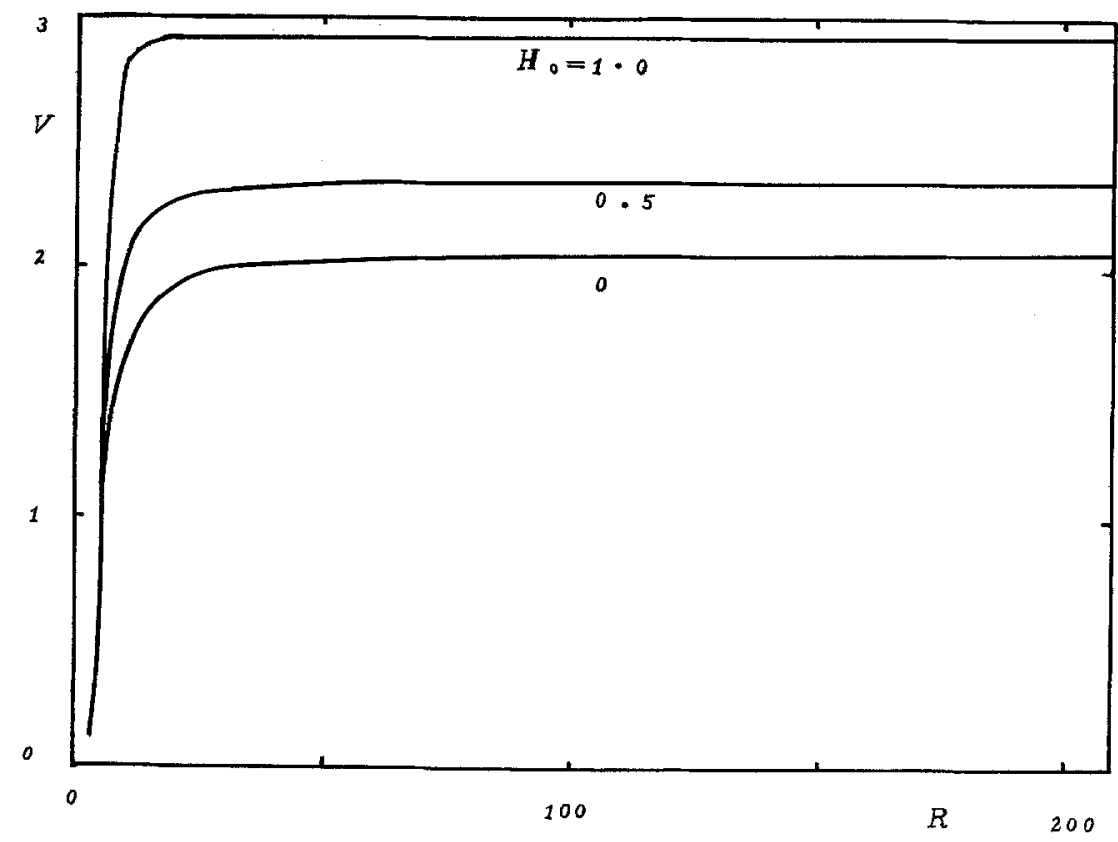

Fig. 4. The distribution of velocity, where $G_{1}=2$ and $Q_{0}=15$.

increment of the Mach number is faster than the increment of $H_{0}$, in this case, the influence of momentum addition produces a larger increment of velocity and a relative small increment of the temperature, and the plasma will be relatively cooler in the case of larger momentum addition. If the parameter $H_{0}$ is large enough, the Mach number will increase rapidly at some place, such as shown in Figure 6. At this point, the 


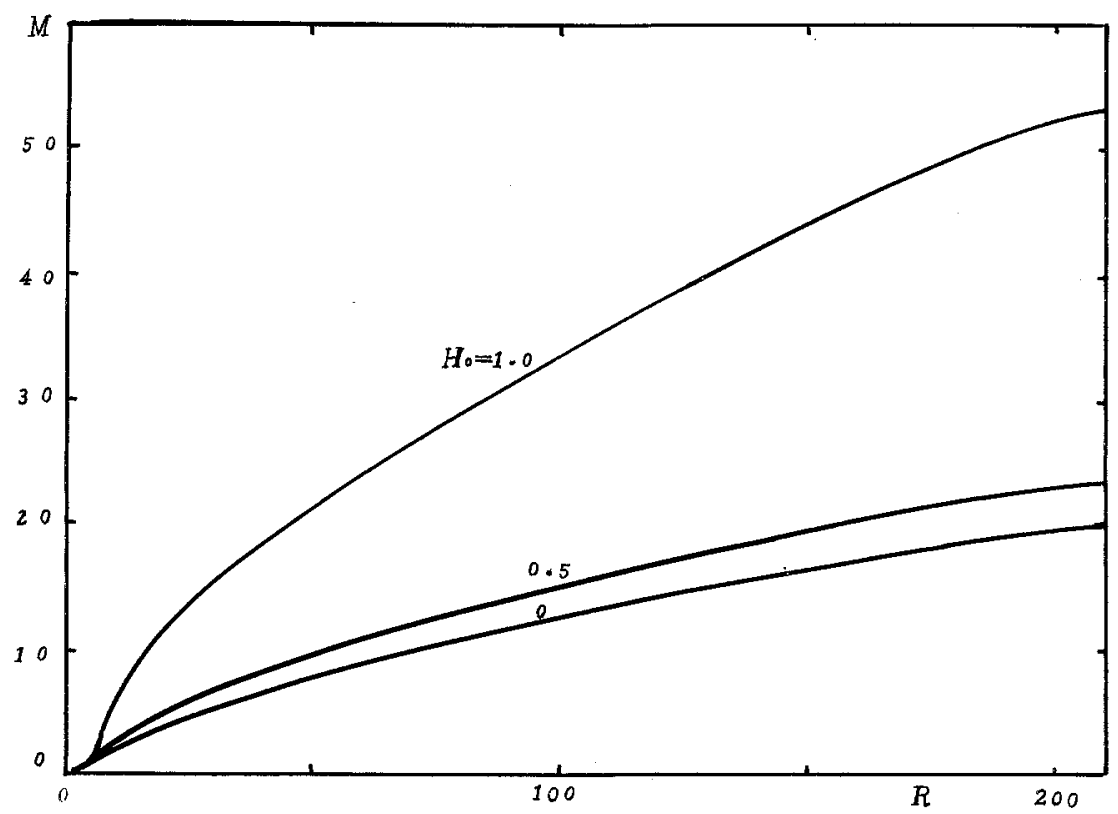

Fig. 5. The Mach number distribution, where $G_{1}=2$ and $Q_{0}=50$.

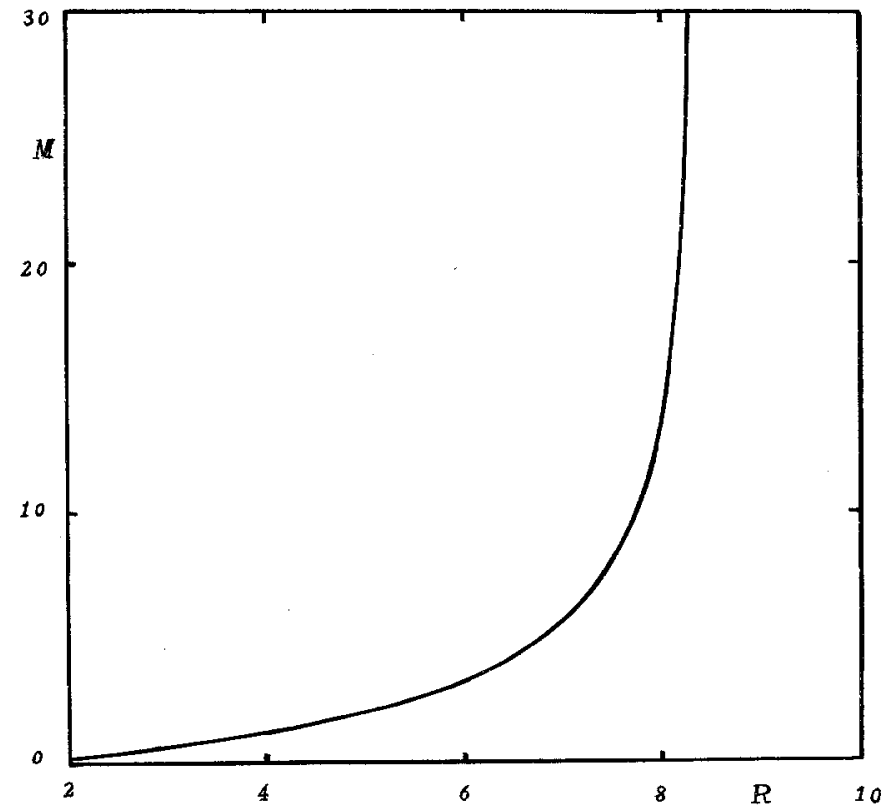

Fig. 6. An example of the Mach number distribution which increases rapidly. The parameters are $G_{1}=6.2577, Q_{0}=10$, and $H_{0}=0.5$. 


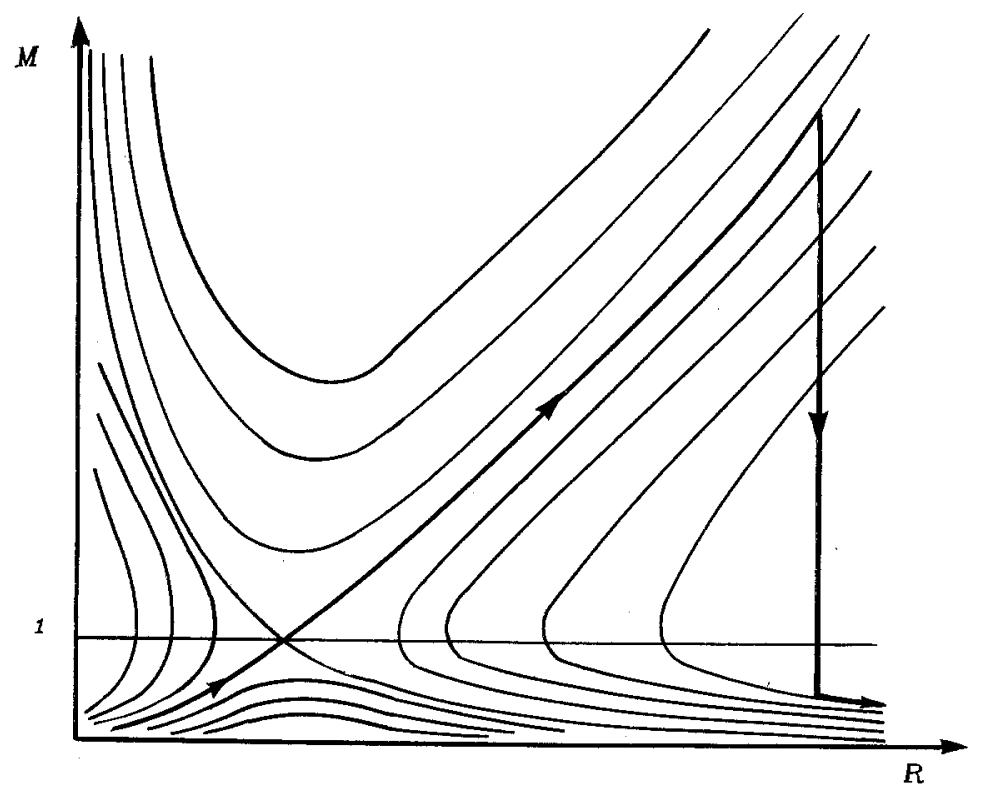

Fig. 7. The integral curves of the solar wind equations, where is a shock jump shown as the thickened line.

temperature decreases rapidly, a heat such as the energy released from recombination is needed, otherwise the cross section of the flux tube will shrink, and there is a shock wave similar to the phenomenon of shock-wave disk in the supersonic wind tunnel. The solution with shock wave corresponds to the discontinuous jump from a supersonic flow to a subsonic flow as shown in Figure 7.

Figure 8 gives the velocity and Mach-number distributions for the parameters $Q_{0}=100, H_{0}=1$, and $G_{1}=2$. Comparing these results with those in Figures 4 and 5 , it shows that the more heat is added, the smaller the velocity and the Mach number at $1 \mathrm{AU}$ are obtained. In fact, the heat energy added in the solar coronal converts partly into kinetic energy and partly into internal energy of the solar wind plasma. As the amount of the energy addition is increased, the position of critical sonic point $R_{s}$ and the plasma temperature in the solar atmosphere will increase also. In this case, the temperature and thus the sonic velocity will increase at the Earth's orbit, on the other hand, the velocity of the solar wind will decrease.

Leer and Holzer (1980) treated the solar wind flow in considerations of the influence of the source or sink of energy and momentum additions by a computational approach. They concluded that the energy or momentum additions in the region of subsonic flow increases the solar wind mass flux but has little effect on or significantly reduced, the solar wind speed at $1 \mathrm{AU}$. The present paper, on the other hand, shows that the velocity of the solar wind at $1 \mathrm{AU}$ can be increased by the momentum addition even in the subsonic flow region. The different conclusions come from the different initial and boundary conditions. We do not impose the condition that the initial mass flux at the initial position $r_{0}$ should keep constant. The implication of this condition can be seen 


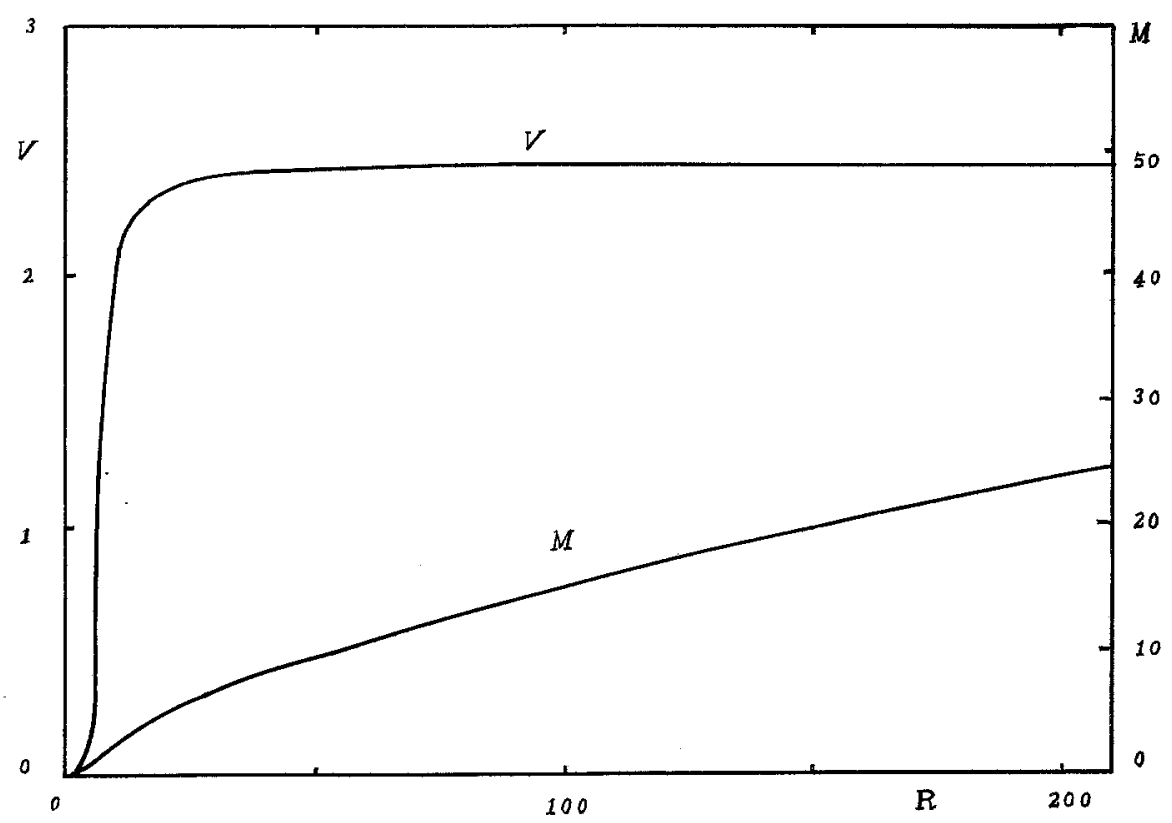

Fig. 8. The distributions of the Mach number and velocity, where $G_{1}=2, Q_{0}=100$, and $H_{0}=1$.

clearly from the condition of mass conservation. Equation (2.3) requires that

$$
\frac{A(1 \mathrm{AU})}{A_{0}}=\frac{\rho_{0} v_{r 0}}{\left(\rho v_{r}\right)_{r=1 \mathrm{AU}}} .
$$

If the initial mass flux $\rho_{0} v_{r 0}$ is fixed, différent value of mass flux at $1 \mathrm{AU}\left(\rho v_{r}\right)_{r=1 \mathrm{AU}}$ requires different value of the ratio of cross-section areas of the flux tube at $1 \mathrm{AU}$ and at initial position $r_{0}$, and these conclusions are obtained for different flux tubes which may have similar configurations. However, the results of the present paper are applied to one, and only one, flux tube. Figures 2, 4, and 8 give the non-dimensional velocity profiles. If the plasma density and temperature at the critical point are same values for different $H_{0}$, the larger mass flux at $1 \mathrm{AU}$ requires a larger mass flux at the initial boundary $r_{0}$. However, the results displayed in the figures show that the relative increment of the mass flux is only about $30 \%$, and the increment may even smaller if the plasma temperature and density at the sonic point are lower or that the distribution of the momentum addition function $g(R)$ in (4.3) is increased first and then decreased. Thus, the momentum addition will not change the mass flux appreciably, but increase the solar wind velocity obviously. Therefore, the momentum addition may be one of the more effective agents for the acceleration of the solar wind and stellar wind. 


\section{Discussion}

The velocity of the solar wind may be obviously increased even if the momentum addition is supplied in the region of subsonic flow region. We suggest that the momentum addition may be the average or fluctuation component of azimuthal magnetic pressure. However, the momentum addition discussed here may has a broader meaning, encompassing other than the magnetic field. Therefore, the conclusions of the present paper is more general, that is, the momentum addition accelerates the solar wind in a fixed streamtube, the larger the momentum addition, the higher the stream velocity. As the conclusion is for a fixed tube, the increase in velocity associates with the increase of the initial mass flux, however, the increment is not large to contradict with the observations. On the other hand, the comparison of different cases in the same streamtube is reasonable.

In the definition of the non-dimensional quantities in relationships (4.2), the typical values are mostly adopted as the ones at the sonic point. This approach is convenient for solving the equations, but they will change if the initial conditions are changed. For example, $G_{1}$ will be smaller if the temperature at sonic point is higher. In Figures 4 and 5 , we use $G_{1}=2$, which is smaller than the value given in Figures 2 and 3 . Comparing these results, it shows that the wind flow is faster in the case of smaller $G_{1}$, which may be relevant to the stellar wind. For examples, the young stars have higher surface temperature and larger mass, the red giant and supergiant have larger radii, the value of $G_{1}$ may be smaller, and thus, the stellar wind may has higher velocity.

The results of the present paper show that the acceleration process is more effective in the open magnetic flux tube if there is an azimuthal magnetic field. According to this concept, there must be higher speed stream from the coronal holes and the polar region. The velocity may increase by about $40 \%$ for a reasonable magnitude of the azimuthal magnetic field, and this may be one of the reasons that the solar wind velocity from the coronal hole is larger than that of the normal solar wind velocity. One of the other reasons may be the larger energy addition in the coronal hole than elsewhere $(\mathrm{Hu}$, 1982a). The velocity of the high-stream flow of the solar wind may be two times the velocity of the normal solar wind if both the momentum and the energy additions are included. This conclusion is useful in explanation the observation of the high-stream flow.

Similar considerations may be applied to the acceleration processes of the stellar wind and the jet beam of double sources in radio galaxy. We cannot be sure whether the stellar atmosphere have structures similar to those of a coronal hole, but it is probable that the stellar, like the solar, magnetic field is nearly a dipole field and the magnetic field near the polar region is open. On the other hand, the observations show the existence of the longitudinal and azimuthal magnetic fields. The acceleration mechanism by momentum, and also energy, additions will be useful in many astrophysical gasdynamical processes. 


\section{Appendix}

In spherical coordinates $(r, \theta, \phi)$, in the case of the axisymmetric condition $\partial / \partial \phi=0$, the magnetohydrodynamic equations may be written in the component forms as follows:

$$
\begin{aligned}
& \frac{1}{r} \frac{\partial \rho v_{r} r^{2}}{\partial r}+\frac{1}{\sin \theta} \frac{\partial \rho v_{r} \sin \theta}{\partial \theta}=0, \\
& \rho\left(v_{r} \frac{\partial v_{r}}{\partial r}+\frac{v_{\theta}}{r} \frac{\partial v_{r}}{\partial \theta}-\frac{v_{\theta}^{2}+v_{\phi}^{2}}{r}\right)=-\frac{\partial p}{\partial r}+\frac{1}{4 \pi}\left(\frac{B_{\theta}}{r} \frac{\partial B_{r}}{\partial \theta}-\frac{B_{\theta}^{2}+B_{\phi}^{2}}{r}\right)- \\
& -\frac{1}{8 \pi} \frac{\partial\left(B_{\theta}^{2}+B_{\phi}^{2}\right)}{\partial r}-\rho \frac{G \cdot \mathscr{M}}{r^{2}}, \\
& \rho\left(v_{r} \frac{\partial v_{\theta}}{\partial r}+\frac{v_{\theta}}{r} \frac{\partial v_{\theta}}{\partial \theta}+\frac{v_{r} v_{\theta}}{r}-\frac{v_{\phi}^{2} \operatorname{ctg} \theta}{r}\right)=-\frac{1}{r} \frac{\partial p}{\partial \theta}+ \\
& +\frac{1}{4 \pi}\left(B_{r} \frac{\partial B_{\theta}}{\partial r}+\frac{B_{r} B_{\theta}}{r}-\frac{B_{\phi}^{2} \operatorname{ctg} \theta}{r}\right)-\frac{1}{8 \pi r} \frac{\partial\left(B_{r}^{2}+B_{\phi}^{2}\right)}{\partial \theta}, \\
& \rho\left(v_{r} \frac{\partial v_{\phi}}{\partial r}+\frac{v_{\theta}}{r} \frac{\partial v_{\phi}}{\partial \theta}+\frac{v_{r} v_{\phi}}{r}+\frac{v_{\theta} v_{\phi} \operatorname{ctg} \theta}{r}\right)=\frac{1}{4 \pi} \times \\
& \times\left(B_{r} \frac{\partial B_{\phi}}{\partial r}+\frac{B_{\theta}}{r} \frac{\partial B_{\phi}}{\partial \theta}+\frac{B_{r} B_{\phi}}{r}+\frac{B_{\theta} B_{\phi} \operatorname{ctg} \theta}{r}\right), \\
& \rho\left(v_{r} \frac{\partial e}{\partial r}+\frac{v_{\theta}}{r} \frac{\partial e}{\partial \theta}\right)-\frac{p}{\rho}\left(v_{r} \frac{\partial \rho}{\partial r}+\frac{v_{\theta}}{r} \frac{\partial p}{\partial \theta}\right)=\frac{1}{r^{2}} \frac{\mathrm{d} r^{2} q}{\mathrm{~d} r}, \\
& \frac{\partial}{\partial \theta}\left[\sin \theta\left(v_{r} B_{\theta}-v_{\theta} B_{r}\right)\right]=0, \\
& \frac{\partial}{\partial r}\left[r\left(v_{r} B_{\theta}-v_{\theta} B_{r}\right)\right]=0, \\
& \frac{\partial}{\partial r}\left[r\left(v_{\phi} B_{r}-v_{r} B_{\phi}\right)\right]-\frac{\partial}{\partial \theta}\left[v_{\theta} B_{\phi}-v_{\phi} B_{\theta}\right]=0, \\
& p=\rho \mathscr{R} T, \\
& \frac{1}{r} \frac{\partial B_{r} r^{2}}{\partial r}+\frac{1}{\sin \theta} \frac{\partial B_{\theta} \sin \theta}{\partial \theta}=0,
\end{aligned}
$$

where the heat flux is assumed to be a function of $r$ only, $\mathbf{q}=q(r) \mathbf{e}_{r}$. It is difficult to obtain the complet solution of such equations. If our interesting is in the local region, the expansion for small angle $\theta-\theta_{0}$ may be used $(\mathrm{Hu}, 1981,1982)$. For example, we 
expand the quantities in the series of angle $\theta$

$$
\begin{aligned}
& \mathbf{v}=\sum_{n=0}^{\infty} \mathbf{v}^{(n)} \theta^{n}, \quad \mathbf{B}=\sum_{n=0}^{\infty} \mathbf{B}^{(n)} \theta^{n}, \quad p=\sum_{n=0}^{\infty} p^{(n)} \theta^{n}, \\
& \rho=\sum_{n=0}^{\infty} \rho^{(n)} \theta^{n}, \quad e=\sum_{n=0}^{\infty} e^{(n)} \theta^{n}, \quad \cdot T=\sum_{n=0}^{\infty} T^{(n)} \theta^{n}
\end{aligned}
$$

When we substitute the series (A.11) into Equations (A.1)-(A.10), equations may be obtained for every order of $\theta^{n}$.

After some calculations, the equations of zero order are:

$$
\begin{aligned}
& v_{\theta}^{(0)}=0, \\
& B_{\theta}^{(0)}=0, \\
& \rho^{(0)}\left(v_{r}^{(0)} \frac{\mathrm{d} v_{r}^{(0)}}{\mathrm{d} r}-\frac{v_{\phi}^{(0) 2}}{r}\right)=-\frac{1}{4 \pi} \times \\
& \rho^{(0)} v_{\phi}^{(0) 2}=\frac{B_{\phi}^{(0) 2}}{4 \pi}, \quad \times\left(B_{\phi}^{(0)} \frac{\mathrm{d} B_{\phi}^{(0)}}{\mathrm{d} r}+\frac{B_{\phi}^{(0) 2}}{r}\right)-\frac{\mathrm{d} p^{(0)}}{\mathrm{d} r}-\rho^{(0)} \frac{G \mathscr{M}}{r^{2}} \\
& \rho^{(0)} v_{r}^{(0)} \frac{\mathrm{d} r v_{\phi}^{(0)}}{\mathrm{d} r}=\frac{B_{r}^{(0)}}{4 \pi} \frac{\mathrm{d} r B_{\phi}^{(0)}}{\mathrm{d} r}, \\
& \rho^{(0)}\left[v_{r}^{(0)} \frac{\mathrm{d} e^{(0)}}{\mathrm{d} r}+v_{r}^{(0)} p^{(0)} \frac{\mathrm{d}}{\mathrm{d} r}\left(\frac{1}{\rho^{(0)}}\right)\right]=\frac{1}{r^{2}} \frac{\mathrm{d} r^{2} q}{\mathrm{~d} r}, \\
& \frac{\mathrm{d}}{\mathrm{d} r}\left[r\left(v_{\phi}^{(0)} B_{r}^{(0)}-v_{r}^{(0)} B_{\phi}^{(0)}\right)\right]-\left[v_{\theta}^{(1)} B_{\phi}^{(0)}-v_{\phi}^{(0)} B_{\theta}^{(1)}\right]=0, \\
& p^{(0)}=\rho^{(0)} \mathscr{R} T^{(0)} \cdot
\end{aligned}
$$

By using Equation (A.15), Equation (A.14) becomes

$$
\rho^{(0)} v_{r}^{(0)} \frac{\mathrm{d} v_{r}^{(0)}}{\mathrm{d} r}=-\frac{\mathrm{d}}{\mathrm{d} r}\left(\frac{B_{\phi}^{(0) 2}}{8 \pi}\right)-\frac{\mathrm{d} p^{(0)}}{\mathrm{d} r}-\rho^{(0)} \frac{G \mathscr{M}}{r^{2}} .
$$

These equations are the basic equations of one-dimensional solar wind flow.

Similarly, the first-order equations may be written as

$$
\frac{1}{r} \frac{\mathrm{d} r^{2} \rho^{(0)} v_{r}^{(0)}}{\mathrm{d} r}+2 \rho^{(0)} v_{\theta}^{(1)}=0,
$$




$$
\begin{aligned}
& \rho^{(0)}\left(v_{r}^{(0)} \frac{\mathrm{d} v_{r}^{(1)}}{\mathrm{d} r}+v_{r}^{(1)} \frac{\mathrm{d} v_{r}^{(0)}}{\mathrm{d} r}+\frac{v_{\theta}^{(1)} v_{r}^{(1)}}{r}-\frac{2}{r} v_{\phi}^{(0)} v_{\phi}^{(1)}\right)+\rho^{(1)}\left(v_{r}^{(0)} \frac{\mathrm{d} v_{r}^{(0)}}{\mathrm{d} r}-\frac{v_{\phi}^{(0) 2}}{r}\right)= \\
& =\frac{1}{4 \pi r}\left(B_{\theta}^{(1)} B_{r}^{(1)}-B_{\phi}^{(0)} \frac{\mathrm{d} r B_{\phi}^{(1)}}{\mathrm{d} r}-B_{\phi}^{(1)} \frac{\mathrm{d} r B_{\phi}^{(0)}}{\mathrm{d} r}\right)-\frac{\mathrm{d} p^{(1)}}{\mathrm{d} r}-\rho^{(1)} \frac{G \mathscr{M}}{r^{2}} \\
& -\frac{2 \rho^{(0)} v_{\phi}^{(0)} v_{\phi}^{(1)}}{r}-\frac{\rho^{(1)} v_{\phi}^{(0) 2}}{r}=\frac{1}{4 \pi r}\left[B_{r}^{(0)}\left(\frac{\mathrm{d} r B_{\theta}^{(1)}}{\mathrm{d} r}-B_{\phi}^{(1)}\right)-3 B_{\phi}^{(0)} B_{\phi}^{(1)}\right]-\frac{p^{(1)}}{r}, \\
& \rho^{(0)} v_{\phi}^{(0)} v_{\theta}^{(1)}+\left(\rho^{(0)} v_{r}^{(1)}+\rho^{(1)} v_{r}^{(0)}\right) \frac{\mathrm{d} r v_{\phi}^{(0)}}{\mathrm{d} r}=\frac{1}{4 \pi} \times \\
& \times\left(B_{\theta}^{(1)} B_{\phi}^{(0)}+B_{r}^{(0)} \frac{\mathrm{d} r B_{\phi}^{(1)}}{\mathrm{d} r}+B_{r}^{(1)} \frac{\mathrm{d} r B_{\phi}^{(0)}}{\mathrm{d} r}\right) \\
& \rho^{(0)}\left(v_{r}^{(0)} \frac{\mathrm{d} e^{(1)}}{\mathrm{d} r}+v_{r}^{(1)} \frac{\mathrm{d} e^{(0)}}{\mathrm{d} r}+\frac{v^{(1)} v_{\phi}^{(0)}}{r}\right)+\rho^{(1)} v_{r}^{(0)} \frac{\mathrm{d} e^{(0)}}{\mathrm{d} r}+ \\
& +\left(\rho^{(0)} p^{(1)}+\rho^{(1)} p^{(0)}\right) v_{r}^{(0)} \frac{\mathrm{d}}{\mathrm{d} r}\left(\frac{1}{\rho^{(0)}}\right)+\rho^{(0)} p^{(0)} \times \\
& \times\left[-v_{r}^{(0)} \frac{\mathrm{d}}{\mathrm{d} r}\left(\frac{\rho^{(1)}}{\rho^{(0) 2}}\right)+v_{r}^{(1)} \frac{\mathrm{d}}{\mathrm{d} r}\left(\frac{1}{\rho^{(0)}}\right)-\frac{v_{\theta}^{(1)}}{r} \frac{\rho^{(1)}}{\rho^{(0) 2}}\right]=0, \\
& v_{r}^{(0)} B_{\theta}^{(1)}=v_{\theta}^{(1)} B_{r}^{(0)}, \\
& \frac{\mathrm{d}}{\mathrm{d} r}\left[r\left(v_{\phi}^{(0)} B_{r}^{(1)}-v_{\phi}^{(1)} B_{r}^{(0)}\right)-r\left(v_{r}^{(0)} B_{\phi}^{(1)}-v_{r}^{(1)} B_{\phi}^{(0)}\right)\right]- \\
& -2\left(v_{\theta}^{(1)} B_{\phi}^{(0)}-v_{\theta}^{(0)} B_{\phi}^{(1)}\right)-\left(v_{\phi}^{(0)} B_{\theta}^{(2)}-v_{\phi}^{(1)} B_{\theta}^{(1)}\right)=0, \\
& \frac{1}{r} \frac{\mathrm{d} r^{2} B_{r}^{(0)}}{\mathrm{d} r}+2 B_{\theta}^{(1)}=0, \\
& p^{(1)}=\mathscr{R}\left(\rho^{(0)} T^{(1)}+\rho^{(1)} T^{(0)}\right) .
\end{aligned}
$$

Similarly, equations may be given for the higher order.

If the basic flow of the solar wind in the flux tube is a spherical expansion, it gives

$$
\rho^{(0)} v_{r}^{(0)} r^{2}=m \text { (constant) . }
$$

Using (A.30), we find that Equation (A.21) gives

$$
v_{\theta}^{(1)}=0 \text {, }
$$


and Equation (A.28) gives

$$
B_{\theta}^{(1)}=0 \text {. }
$$

Substituting (A.32) into (A.28), the condition of magnetic flux conservation is

$$
B_{r}^{(0)} r^{2}=\Phi(\text { constant) }
$$

As the plasma and magnetic field are frozen together, conditions (A.30) and (A.33) are consisted.

\section{References}

Belcher, J. W.: 1971, Astrophys. J. 168, 509.

Bridle, A. H.: 1982, in D. S. Heeschen and C. M. Wade (eds.), Extragalactic Radio Sources, D. Reidel Publ. Co., Dordrecht, Holland, p. 121.

Hollweg, J. V.: 1973, Astrophys. J. 181, 547.

Holzer, T. E.: 1977, J. Geophys. Res. 82, 23.

Holzer, T. E.: 1977, in L. J. Lanzerotti, C. F. Kennel, and E. N. Parker (eds.), Solar System Plasma Physics, Vol. 1, North-Holland Publ. Co., Amsterdam, p. 105.

Hu, W. R.: 1981a, Acta Space Sci. Sinica 1, 90.

Hu, W. R.: 1981b, Scientia Sinica 24, 1680.

Hu, W. R.: 1981c, Acta Astrophys. Sinica 1, 222.

Hu, W. R.: 1982a, Geophys. Astrophys. Fluid Dynamics 19, 311.

Hu, W. R.: 1982b, Scientia Sinica 25, 511.

Hundhausen, A. G.: 1972, Coronal Expansion and Solar Wind, Springer Verlag, Berlin.

Leer, E. and Holzer, T. E.: 1980, J. Geophys. Res. 85, 4681.

Parker, E. N.: 1963, Interplanetary Dynamical Processes, Interscience Publ., New York.

Pneuman, G. W.: 1971, Solar Phys. 18, 255.

Whang, Y. C. and Chien, T. H.: 1978, Astrophys. J. 221, 350.

Weber, E. J. and Davis, L.: 1967, Astrophys. J. 148, 217.

Yeh, T. and Pneuman, G. W.: 1976, Solar Phys. 54, 419. 